\title{
Motivación hacia la clase de educación física en preadolescentes mexicanos y españoles Motivation towards physical education class in Mexican and Spanish preadolescents
}

*Humberto Blanco Vega, **Daniel Mayorga-Vega, *José René Blanco Ornelas, *Jesús Enrique Peinado Pérez, *Perla Jannet Jurado García

*Universidad de Chihuahua (México), **Universidad de Jaén (España)

Resumen. El objetivo de esta investigación consistió en comparar los perfiles de motivación hacia las clases de educación física de niños y niñas mexicanos y españoles. La muestra total fue de 606 sujetos; 306 españoles (139 mujeres y 167 hombres) y 300 mexicanos (166 mujeres y 134 hombres), con una edad media de 10.70 años (DE= 0.71). El abordaje adoptado en la investigación se enmarcó dentro de un enfoque cuantitativo con un diseño descriptivo tipo encuesta. En los resultados del análisis multivariante de la varianza, seguido por los análisis de varianza univariados se encontró que, los preadolescentes españoles en relación a los preadolescentes mexicanos mostraron mayores niveles de motivación en las clases de educación física, así como en su regulación integrada e identificada, además presentaron menores niveles de desmotivación; mientras que, en los preadolescentes mexicanos se encontraron mayores niveles de regulación introyectada y regulación externa así como mayores niveles de desmotivación en relación con los adolescentes españoles. Futuras investigaciones deberían replicar estos hallazgos en muestras más amplias.

Palabras clave: motivación, clase de educación física, autoregulación, locus, nacionalidad.

Abstract. The aim of this study was to compare the motivation profiles for physical education classes of Mexican and Spanish children. The total sample was 606 subjects; 306 Spaniards (139 girls and 167 boys) and 300 Mexicans (166 girls and 134 boys), with an average age of 10.70 years $(\mathrm{SD}=0.71)$. The approach adopted in the research was framed within a quantitative approach with a descriptive design as a type as a survey. In the results of the multivariate analysis of variance, followed by the univariate analysis of variance, it was found that Spanish preadolescents in relation to Mexican preadolescents showed higher levels of motivation in physical education classes, as well as in their integrated and identified regulation, also presented lower levels of demotivation; whereas, in Mexican preteens, higher levels of introjected regulation and external regulation were found, as well as higher levels of demotivation in relation to Spanish adolescents. Future research should replicate these findings in larger samples.

Keywords: motivation, physical education classes, self-regulation, locus, nationality.

\section{Introducción}

La actividad física es fundamental para el ser humano, imprescindible para su formación y buena salud (García \& Fonseca, 2012), como es sabido la actividad física genera grandes beneficios, tales como la disminución de factores de riesgo en enfermedades cardíacas, niveles de presión, índices de colesterol, síndrome metabólico, riesgo de depresión; asimismo, mejora el tono muscular, mantiene las habilidades de pensamiento y aprendizaje, calidad del sueño, entre otras (Division of Nutrition Physical Activity and Obesity National Center for Chronic Disease Prevention and Health Promotion, 2018; Vidarte, Vélez, Sandoval \& Alfonso, 2011). Además, la práctica de actividad física en los adolescentes mejora el autoconcepto físico y su percepción social (Martínez \& González, 2017).

Por otra parte, la educación física tiene como objetivo promover el compromiso de los adolescentes y el apoyo a la actividad física y deportiva del tiempo libre (Jaakkola, Washington \& Yli-Piipari, 2013); así como ofrecer a los estudiantes oportunidades para mejorar sus relaciones, cooperación y nivel de responsabilidad, además de sus habilidades; por lo que, es relevante comprender los diferentes factores personales y situacionales que afectan la motivación de los estudiantes adolescentes y profundizar en el análisis de su voluntad de éxito, dado el impacto que puede tener la actividad física en ellos (Baena, Ruiz-Juan \& Granero-Gallegos, 2016; Menéndez \& Fernández-Río, 2017).

Fecha recepción: 03-09-18. Fecha de aceptación: 01-02-19

Perla Jannet Jurado Garcí

pjurado@uach.mx
Por otro lado, tomando en cuenta la revisión de Owen, Smith, Lubans, Ng y Lonsdale (2014) se puede concluir fehacientemente sobre la existencia de una relación entre la motivación y la actividad física; lo que permite afirmar que la motivación se convierte en un predictor de la persistencia del comportamiento para realizar actividad física (Baena et al., 2016).

Pero, ¿qué es la motivación? el concepto de motivación se ha definido como el constructo hipotético que describe las fuerzas internas y/o externas que producen la iniciativa, dirección, intensidad y persistencia del comportamiento. La motivación puede conducir a importantes consecuencias de al menos tres tipos: afectivo, cognitivo y conductual. El énfasis en las fuerzas internas y externas encaja muy bien con la presencia de dos tipos principales de motivación que han sido muy investigados, a saber, la motivación intrínseca y extrínseca. Se ha definido a la motivación intrínseca como hacer algo por sí mismo; mientras que la motivación extrínseca se refiere a hacer algo como un medio para obtener un fin y no por el propio bien; ambos tipos de motivaciones han sido temas muy populares y han permitido a los investigadores dar sentido a varios fenómenos de la importancia en el deporte y la actividad física (Vallerand, 2007).

Dentro de las teorías motivacionales se encuentra la teoría de la autodeterminación (Deci \& Ryan, 2012) la cual es una teoría derivada empíricamente de la motivación humana y la personalidad en contextos sociales que diferencia la motivación en términos de ser autónomo y controlado; basada en experimentos que examinaban los efectos de las recompensas extrínsecas en la motivación intrínseca. Además han surgido cinco mini-teorías para abordar diferentes problemas, aunque relacionados; los efectos de los entornos 
sociales sobre la motivación intrínseca y la autorregulación a través de la internalización y la integración; las diferencias individuales en las orientaciones motivacionales generales; el funcionamiento de las necesidades psicológicas universales fundamentales que son esenciales para el crecimiento, la integridad y el bienestar; y los efectos de los diferentes contenidos de los objetivos sobre el bienestar y el rendimiento.

En base a dicha teoría se han desarrollado una serie de instrumentos, entre ellos la Escala del Locus Percibido de Causalidad (PLOC) donde estudios basados en la teoría de la autodeterminación han demostrado la existencia de una relación significativa entre la percepción que los alumnos tienen del clima motivacional de la clase de educación física, la motivación intrínseca y las intenciones de ser físicamente activos (Gutiérrez, 2014; Jaakkola et al., 2013).

En este estudio se intenta determinar las diferencias y similitudes entre preadolescentes mexicanos y españoles en cuanto a sus perfiles de motivación hacia las clases de educación física.

En consecuencia, esta investigación pretende, como investigación aplicada, aportar información que se traduzca en una práctica educativa de mayor calidad en el contexto de atención a la diversidad; contribuyendo al saber pedagógico en el esclarecimiento de los factores que conforman un modelo de desarrollo humano integral; bajo la premisa de que los esfuerzos educativos deben enfocarse hacia el aumento de los sentimientos de autovaloración y competencia de los estudiantes, fortaleciendo la autoestima y el autoconcepto, lo que a su vez favorecerá la motivación hacia el logro, las relaciones interpersonales y en general la forma particular de desenvolverse frente a diversas tareas y desafíos que se les presenten.

\section{Metodología}

\section{Participantes}

Participaron en el estudio 606 preadolescentes, 306 españoles (139 mujeres y 167 hombres) y 300 mexicanos (166 mujeres y 134 hombres). La edad de los participantes fluctuó entre los 10 y 12 años, con una media de 10.70 y una desviación estándar de 0.71 años.

\section{Instrumento}

Para medir los perfiles de motivación hacia las clases de educación física se utilizó la Escala de Locus Percibido de Causalidad con la regulación integrada (PLOC-2) adaptada por Ferriz, González-Cutre y Sicilia (2015) la cual consta de 6 factores con 4 ítems cada uno, que miden la motivación intrínseca, la regulación integrada, la regulación identificada, la regulación introyectada, la regulación externa y la desmotivación. El instrumento utiliza una escala tipo Likert de 1 (totalmente en desacuerdo) a 7 (totalmente de acuerdo).

\section{Diseño}

En cuanto al diseño del estudio, se utilizó un enfoque cuantitativo con un diseño descriptivo y transversal tipo encuesta (Hernández, Fernández \& Baptista, 2014). Las variables independientes fueron la nacionalidad (mexicanos y españoles) y el género, y las variables dependientes el pro- medio de las puntuaciones en los factores motivación intrínseca, regulación integrada, regulación identificada, regulación introyectada, regulación externa y desmotivación del cuestionario Locus Percibido de Causalidad.

\section{Procedimiento}

Una vez conseguido el permiso tanto de las autoridades educativas como el de las familias, se invitó a participar en el estudio a niños y niñas estudiantes de la ciudad de Chihuahua, México y de la ciudad de Granada, España. Los que aceptaron participar firmaron el consentimiento informado. Luego se aplicó el instrumento antes descrito en una sesión de aproximadamente 20 minutos; en las aulas de los centros educativos. Al inicio de la evaluación se comentaban las instrucciones del cuestionario y los objetivos de la investigación.

Después de aplicar el instrumento se procedió a recopilar y a analizar los datos mediante el paquete SPSS 20.0.

\section{Análisis estadístico de los datos}

Se realizaron análisis estadísticos descriptivos (medias y desviaciones estándar) para todas las variables dependientes. Posteriormente, después de verificar que los datos cumplían con los supuestos de los análisis estadísticos paramétricos, se llevó a cabo un análisis multivariante de varianza (MANOVA), seguido por los análisis de varianza univariados (ANOVAs), para examinar las diferencias entre niñas y niños mexicanos y españoles en cuanto a su locus percibido de causalidad, el tamaño del efecto se estimó mediante el eta cuadrado $\left(\eta^{2}\right)$. Todos los análisis estadísticos se realizaron con el programa SPSS versión 20.0 para Windows. El nivel de significación estadística se estableció en $p=.05$.

\section{Resultados}

La Tabla 1 muestra los valores de las medias y las desviaciones estándar de la puntuación en los factores de Locus Percibido de Causalidad de acuerdo a las variables naciona-

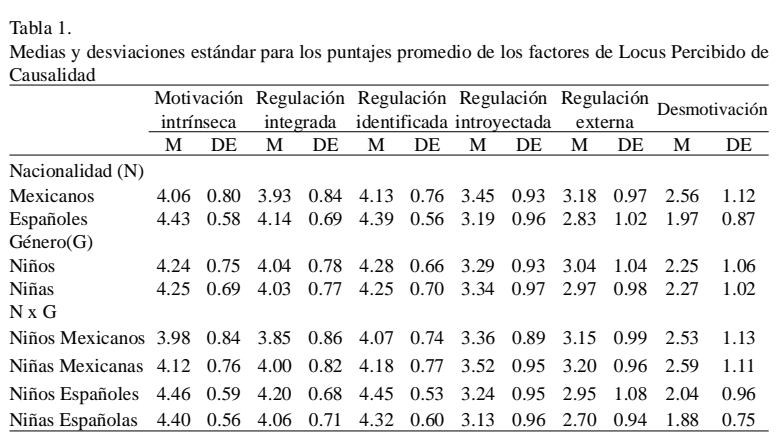

lidad y género.

La Tabla 2 muestra los resultados de los MANOVAs y los subsiguientes ANOVAs. Los resultados de los MANOVAs indicaron diferencias significativas globales de acuerdo a la variable nacionalidad en las puntuaciones de puntajes promedio de los factores de Locus Percibido de Causalidad (Wilks' $\lambda=.847 ; p<.001 ; \eta^{2}=.153$ ). Posteriormente, los resultados de los ANOVAs mostraron que los españoles reportan mayores niveles de motivación intrínse- 
Análisis de varianza multivariante y univariante para los puntajes promedio de los factores de Locus Percibido de Causalidad

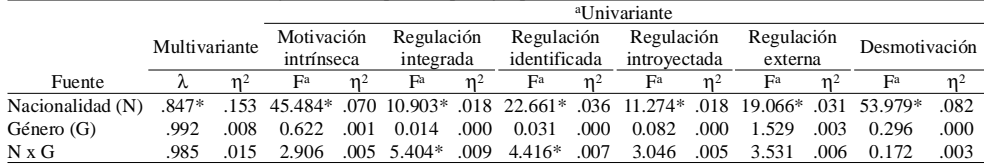
\begin{tabular}{lrrr}
$\mathrm{N} \mathrm{x} \mathrm{G}$ & .985 & .015 & 2.906 \\
\hline Nota: & aUnivariante $\mathrm{gl}=1,602 *=p<.05$
\end{tabular}

ca $(F=45.484, p<.001)$, regulación integrada $(F=10.903, p<$ .001) y regulación identificada $(F=22.661, p<.001)$ mientras que los mexicanos lo hace con respecto a regulación introyectada $(F=11.274, p<.001)$, regulación externa $(F=$ 19.066, $p<.001)$ y desmotivación $(F=53.979, p<.001)$.

\section{Discusión}

El objetivo de este estudio fue determinar las diferencias y similitudes entre preadolescentes mexicanos y españoles en cuanto a sus perfiles de motivación hacia la clase de educación física, vista la importancia que tienen estas metas en el ámbito académico. Este tipo de comparaciones entre países son de gran interés, como bien demuestran los datos publicados por otras investigaciones (Franco, Coterón, Gómez, Brito \& Martínez, 2017; Ruiz-Juan, Ortiz-Camacho, García-Montes, Baena-Extremera \& Baños, 2018; Vílchez, Ruiz-Juan \& García, 2017). Aun cuando se encontraron diferencias estadísticamente significativas en relación a la nacionalidad (españoles y mexicanos), los resultados no concuerdan del todo con los reportados por Ruiz-Juan et al. (2018), en una muestra de adolescentes (11 a 16 años de edad). Ruiz-Juan y colaboradores encontraron que los mexicanos fueron quienes presentaron medias más altas en cada una de las dimensiones del cuestionario utilizado para medir la motivación intrínseca, la regulación integrada, la regulación identificada, la regulación introyectada, la regulación externa y la desmotivación; mientras que en nuestro estudio los preadolescentes españoles fueron quienes obtuvieron medias más altas en motivación intrínseca, regulación integrada e identificada; así mismo, Granero-Gallegos, Baena, Sánchez-Fuentes y Martínez-Molina (2014) reportaron que los adolescentes presentan una alta motivación intrínseca hacia las clases de educación física pero una baja desmotivación, en cambio Sánchez et al. (2017) reportan una alta identificación regulada y una baja desmotivación. Por último, no se encontraron diferencias significativas de acuerdo al género, resultados que, en cuanto al factor desmotivación, concuerdan con los reportados por Gómez, Gámez y Martínez (2011); no obstante es importante continuar analizando los diferentes factores de motivación hacia la clase de educación física y su relación con el género (González-Cutre \& Sicilia, 2012).

\section{Conclusiones}

De los resultados mostrados y de su análisis, se pueden obtener las siguientes conclusiones:

Los preadolescentes españoles en relación a los preadolescentes mexicanos mostraron un mejor perfil de motivación hacia la clase de educación física, con un mayor nivel de motivación intrínseca, regulación integrada e identificada, mientras que en los preadolescentes mexicanos se encontraron mayores niveles de regulación introyectada y regulación externa, así como mayores niveles de desmotivación. De acuerdo al género no se encontraron diferencias estadísticamente significativas.

Las diferencias encontradas entre mexicanos y españoles con respecto a su motivación en la clase de educación física sugieren que al diseñar cualquier tipo de intervención que tenga como objetivo potenciar dicha motivación habrá que tomar en cuenta a la variable nacionalidad; no obstante, es preciso desarrollar más investigación al respecto pues el tema trasciende del todo los alcances de la presente investigación.

Por último, cuando menos dos limitaciones están presentes en este trabajo. La primera es que los participantes son solo estudiantes entre 10 y 12 años, lo que supone una amenaza para la posibilidad de generalizar estos resultados. Ampliar la muestra (agregando por ejemplo estudiantes de otras edades) es un área de trabajo de cara al futuro. La segunda limitación proviene del propio instrumento de medición, que se basa en el autoinforme y que por ello puede contener los sesgos que se derivan de la deseabilidad social.

\section{Agradecimientos}

El presente estudio fue financiado por la Secretaría de Educación Pública-Subsecretaría de Educación Superior-Dirección de Superación Académica-Programa para el Desarrollo Profesional Docente (DE-13-6894), así como la facilidad para la financiación de la publicación (CONACYT, Redes Temáticas, No. 269614). Además, el segundo de los autores recibe una ayuda del Plan Propio del Vicerrectorado de Investigación y Transferencia de la Universidad de Granada.

\section{Referencias}

Baena, A., Ruiz-Juan, F., \& Granero-Gallegos, A. (2016). A cross-cultural analysis in predicting 2x2 achievement goals in physical education based on social goals, perceived locus of causality and causal attribution. Studia Psychologica, 58(1), 74.

Deci, E. L., \& Ryan, R. M. (2012). Self-Determination Theory. En P. A. M. Van Lange, A. W. Kruglanski \& E. T. Higgins (Eds.), Handbook of Theories of Social Psychology: Collection: Volumes 1 \& 2 (pp. 416-437). New Delhi: SAGE Publications.

Division of Nutrition Physical Activity and Obesity National Center for Chronic Disease Prevention and Health Promotion. (2018). Physical Activity and Healt. https:// www.cdc.gov/physicalactivity/basics/pa-health/ index.htm

Ferriz, R., González-Cutre, D., \& Sicilia, Á. (2015). Revisión de la Escala del Locus Percibido de Causalidad (PLOC) para la Inclusión de la Medida de la Regulación Integrada en Educación Física. Revista de Psicología del Deporte, 24(2), 329-338.

Franco, E., Coterón, J., Gómez, V., Brito, J. \& Martínez, H.A. (2017). Influencia de la motivación y del flow disposicional sobre la intención de realizar actividad físico-deportiva 
en adolescentes de cuatro países. RETOS. Nuevas tendencias en Educación Física, Deporte y Recreación (31), 46-51.

García, J., \& Fonseca, C. (2012). La actividad física en los y las jóvenes mexicanos y mexicanas: un análisis comparativo entre las universidades públicas y privadas. MHSalud, 9(2), 1-29.

Gómez, A., Gámez, S., \& Martínez, I. (2011). Efectos del género y la etapa educativa del estudiante sobre la satisfacción y la desmotivación en Educación Física durante la educación obligatoria. Ágora para la Educación Física y el deporte, 13(2), 183-195.

González-Cutre, D., \& Sicilia, Á. (2012). Motivation and Exercise Dependence: A Study Based on SelfDetermination Theory. Research Quarterly for Exercise and Sport, 83(2), 318-329.

Granero-Gallegos, A., Baena, A., Sánchez-Fuentes, J., \& Martínez-Molina, M. (2014). Perfiles motivacionales de apoyo a la autonomía, autodeterminación, satisfacción, importancia de la educación física e intención de práctica física en tiempo libre. Cuadernos de Psicología del Deporte, 14(2), 59-69.

Gutiérrez, M. (2014). Relaciones entre el clima motivacional, las experiencias en educación física y la motivación intrínseca de los alumnos. RETOS. Nuevas tendencias en Educación Física, Deporte y Recreación (26), 9-14.

Hernández, R., Fernández, C., \& Baptista, P. (2014). Metodología de la investigación. México: McGraw- Hill.

Jaakkola, T., Washington, T., \& Yli-Piipari, S. (2013). The association between motivation in school physical education and self-reported physical activity during Finnish junior high school: A self-determination theory approach. European Physical Education Review, 19(1), 127-141. doi: 10.1177/1356336X12465514

Martínez, F., \& González, J. (2017). Autoconcepto, práctica de actividad física y respuesta social en adolescentes.
Relaciones con el rendimiento académico. Revista Iberoamericana de Educación, 73(1), 87-108.

Menéndez, J. I., \& Fernández-Río, J. (2017). Responsabilidad social, necesidades psicológicas básicas, motivación intrínseca y metas de amistad en educación física. RETOS. Nuevas tendencias en Educación Física, Deporte y Recreación (32), 134-139.

Owen, K. B., Smith, J., Lubans, D. R., Ng, J. Y., \& Lonsdale, C. (2014). Self-determined motivation and physical activity in children and adolescents: A systematic review and meta-analysis. Preventive medicine, 67, 270-279. doi: 10.1016/j.ypmed.2014.07.033

Ruiz-Juan, F., Ortiz-Camacho, M. M., García-Montes, M. E., Baena-Extremera, A. \& Baños, R. (2018). Predicción transcultural del clima motivacional en educación física. Revista Internacional de Medicina y Ciencias de la Actividad Física y el Deporte, 18(69), 165-183. doi: 10.15366/rimcafd2018.69.011

Sánchez, M., Lizaso, I., Hermosilla, D., Alcover, C.-M., Goudas, M., \& Arranz-Freijó, E. (2017). Preliminary validation of the Perceived Locus of Causality scale for academic motivation in the context of university studies (PLOC-U). British Journal of Educational Psychology, 87, 558-572. doi: 10.1111/bjep.12164

Vallerand, R. J. (2007). Intrinsic and extrinsic motivation in sport and physical activity: A review and a look at the future. En G. Tenenbaum \& R. C. Eklund(Eds.), Handbook of Sport Psychology (pp. 59-83): Wiley.

Vidarte, J., Vélez, C., Sandoval, C., \& Alfonso, M. (2011). Actividad física: estrategia de promoción de la salud. Revista Hacia la Promoción de la Salud, 16(1), 202-218.

Vílchez, M. P., Ruiz-Juan, F. \& García, M. E. (2017). Estudio transcultural de la percepción de competencia escolar y tiempo de ocio. Revista Internacional de Medicina y Ciencias de la Actividad Física y el Deporte, 17(67), 573-587. doi: 10.15366/rimcafd2017.67.012
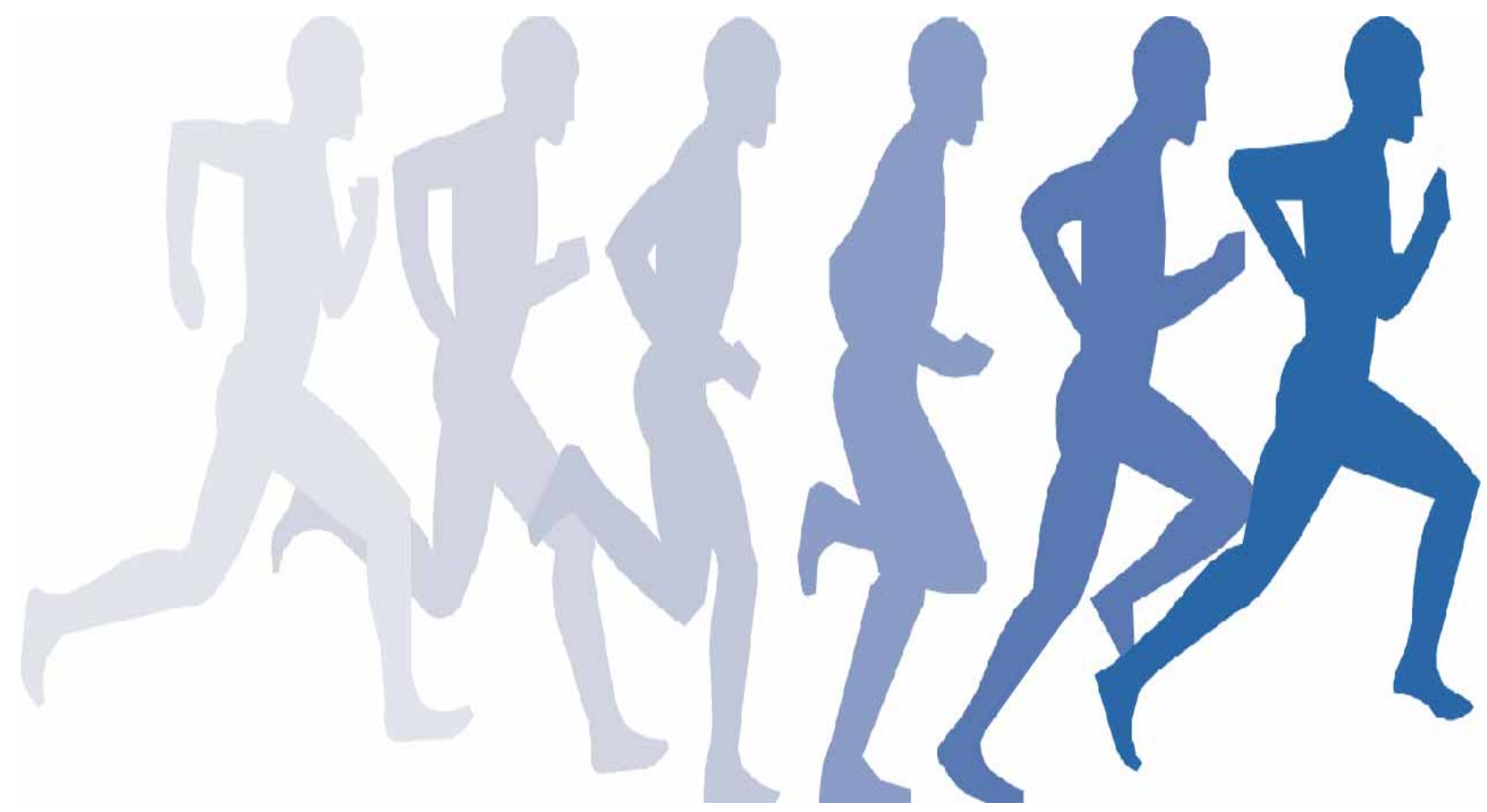